\title{
The challenge of matching assays to biology in DNA damage response biomarkers for response to radiotherapy in bladder cancer
}

\author{
Neil B. Desai ${ }^{1}$, Aditya Bagrodia ${ }^{2}$ \\ ${ }^{1}$ Department of Radiation Oncology, ${ }^{2}$ Department of Urology, University of Texas Southwestern, Dallas, TX, USA \\ Correspondence to: Neil B Desai, MD MHS. Department of Radiation Oncology, University of Texas Southwestern Medical Center, 2800 Inwood Ave. \\ Blvd, Dallas, TX 75390, USA. Email: Neil.Desai@utsouthwestern.edu. \\ Provenance: This is an invited article commissioned by Section Editor Xiao Li (Department of Urology, Jiangsu Cancer Hospital \& Jiangsu Institute \\ of Cancer Research \& Nanjing Medical University Affiliated Cancer Hospital, Nanjing, China). \\ Comment on: Walker AK, Karaszi K, Valentine H, et al. MRE11 as a Predictive Biomarker of Outcome After Radiation Therapy in Bladder Cancer. \\ Int J Radiat Oncol Biol Phys 2019;104:809-18.
}

Submitted Jun 27, 2019. Accepted for publication Jul 09, 2019.

doi: 10.21037/tau.2019.07.05

View this article at: http://dx.doi.org/10.21037/tau.2019.07.05

For the last 40 years of investigations into radiation (RT) for bladder preservation in muscle invasive bladder cancer (MIBC), the best predictor of response has remained early response. Specifically, the ability to achieve complete transurethral resection of bladder tumor (TURBT) prior to treatment (1) and complete or near-complete (Ta/Tis) response following a 4-5 week induction phase each are strongly associated with improved outcome (2).

Despite additional selection criteria (3), a significant residual risk of recurrence and of subsequent increased morbidity of a post-RT salvage cystectomy are key reasons for lack of broader use of bladder preservation strategy. Accordingly, substantial efforts have been directed towards more personalized biomarkers predicting for response to RT in bladder cancer, with multiple groups focusing on canonical DNA damage repair (DDR) pathways (4-8) that classically are thought to mediate a cell's survival in response to ionizing RT.

In a recent issue of the Red fournal, Walker and colleagues (9) attempt to complete the capstones of standardization and validation to a decade of discovery work on the most advanced such DDR biomarker for radiotherapy in bladder cancer, protein expression by immunohistochemistry (IHC) of MRE11, ultimately falling short. MRE11 is a component of the MRN complex atop a central pathway in double-strand break (DSB) repair (10), which is of high relevance to RT response (11).
Previously, the authors first demonstrated an association of low quartile MRE11 IHC expression by semi-quantitative score (median $\%$ positive cells $\times$ modal intensity) with poorer cancer-specific survival, following radiotherapy based bladder preservation in both a test and validation cohort, but not in a cystectomy cohort (5), suggesting it is a predictive marker specifically for RT. While the result that low DDR protein expression would confer worse RT was paradoxical, soon thereafter, Laurberg et al. (6) independently validated the predictive relationship of low quartile MRE11 expression (using identical antibody and scoring system) to poorer cancer-specific survival in primarily chemoradiation treated MIBC patients $(83 \%$ of RT cohort) but not in cystectomy cohorts. Our own study (12) failed to correlate MRE11 IHC with outcome in chemoradiation treated MIBC and further found discordance between two different antibodies, but it was limited by small size and thus inconclusive.

Combined, these data made MRE11 IHC the leading biomarker candidate for RT based bladder preservation in recent years (13). On the heels of this enthusiasm, Walker and colleagues performed a standardization and validation study of MRE11 IHC across multiple centers in the United Kingdom and United States. Disappointingly, despite exhaustive rigor and extensive preparation and adaptation for scoring IHC by this expert group, MRE11 IHC scoring could not be standardized between centers, mainly driven 
by wide variance in scoring of staining intensity between observers. Further, the investigators were unable to reliably re-demonstrate association of MRE11 IHC with outcome, and it was not clear which methodological changes (automated staining, scoring observer training, use of samples from a trial with hypoxia modifier) may have most contributed. Finally, it should be noted that even in prior studies, it was not demonstrated that MRE11 IHC correlated to local recurrence, only to cause-specific survival, leaving a gap in its argument as a RT sensitivity predictor.

\section{Where does this leave us in the effort to better inform on the viability of bladder preservation for an individual patient using biomarkers?}

Unfortunately, MRE11 as assayed by IHC is unlikely to be salvaged a viable biomarker, and skepticism may now accompany use of any other proposed IHC based DDR biomarker. Whether ongoing efforts with automated quantitative analysis (AQUA) focused on an internally controlled nuclear: cytoplasmic ratio of MRE11 (14) or alternative avenues of investigation of MRE11 germline variants or post-transcriptional regulation $(15,16)$ will reinvigorate this line of study is unclear.

However, as the authors note, more traction has been gained of analyzing the relationship of response to cisplatin based chemotherapy in bladder cancer to more reliably assayed somatic DNA alterations in DDR, such as in the nucleotide excision repair (NER) pathway gene ERCC2 (17-19). Robustness of increasingly utilized clinical grade sequencing assays for such alterations compares favorably to that of IHC, and moreover the biologic basis for deficiency in ERCC2 to sensitize to platinum based chemotherapy is more tenable $(17,18)$. Accordingly, study of DDR pathway alterations as biomarkers has been streamlined, with ongoing efforts highlighted by the ongoing the Alliance A031701 phase II trial's (NCT 03609216) assessment of chemotherapy only bladder preservation in patients with deleterious DDR alterations achieving near complete response to neoadjuvant cisplatin based chemotherapy.

Not to be left behind, improved response to chemoradiation also has been associated in our own work with deleterious alterations in DDR pathway genes (12), most commonly in ERCC2. It is easy to speculate that there exists overlap in biomarkers that may predict for improved response to cytotoxic stresses as similar as chemoradiation and neoadjuvant chemotherapy, especially when utilizing common agents. However, significant challenges to study remain here as well: tumor heterogeneity, lack of consensus on what defines a 'deleterious' DDR alteration or DDR genes, deciphering contribution of TURBT $v s$. radiosensitizing chemotherapy $v s$. radiotherapy to outcome, controlling for a wide variance in radiosensitizing chemotherapy regimens, and requirement for large contemporary validation sets when bladder preservation therapy remains infrequently used.

Finally, with the introduction of immune checkpoint inhibition (ICI) into the localized bladder cancer perioperative and post-chemoradiation space (SWOG/NRG 1806, NCT 03775265) there exists a new need to discern predictors of response to immuno-chemoradiation. While data are conflicting on whether DDR alterations or its proposed surrogate somatic mutation burden are associated with ICI response in the advanced bladder cancer setting (20), study in this new context of combination with chemoradiation is eagerly awaited.

\section{What do we do while we await such biomarkers?}

In the interim, it should be stressed that patients appropriately selected by traditional factors for bladder preservation do in fact achieve high rates of complete response and long term, prospective trial based outcomes comparable to cystectomy series (3). Other studies further have shown success with a flexible array of chemoradiation regimens adaptable to patient co-morbidity, emphasized a significant competing risk of distant relapse which should humble arguments for local therapy escalation to cystectomy at all costs in an often heavily co-morbid elderly population, and demonstrated the feasibility of required surveillance and salvage cystectomy (21). While personalized biomarkers of outcome would add value, in the current environment of under-utilization of chemoradiation for MIBC (22), the simplest predictor of bladder preservation is whether one is offered bladder preservation.

\section{Acknowledgments}

The authors would like to thank the Dedman Family Clinical Scholars program for support.

\section{Footnote}

Conflicts of Interest: The authors have no conflicts of interest to declare.

Ethical Statement: The authors are accountable for all 
aspects of the work in ensuring that questions related to the accuracy or integrity of any part of the work are appropriately investigated and resolved.

\section{References}

1. Efstathiou JA, Spiegel DY, Shipley WU, et al. Long-term outcomes of selective bladder preservation by combinedmodality therapy for invasive bladder cancer: the MGH experience. Eur Urol 2012;61:705-11.

2. Mitin T, George A, Zietman AL, et al. Long-Term Outcomes Among Patients Who Achieve Complete or Near-Complete Responses After the Induction Phase of Bladder-Preserving Combined-Modality Therapy for Muscle-Invasive Bladder Cancer: A Pooled Analysis of NRG Oncology/RTOG 9906 and 0233. Int J Radiat Oncol Biol Phys 2016;94:67-74.

3. Chen RC, Shipley WU, Efstathiou JA, et al. Trimodality bladder preservation therapy for muscle-invasive bladder cancer. J Natl Compr Canc Netw 2013;11:952-60.

4. Kawashima A, Nakayama M, Kakuta Y, et al. Excision repair cross-complementing group 1 may predict the efficacy of chemoradiation therapy for muscle-invasive bladder cancer. Clin Cancer Res 2011;17:2561-9.

5. Choudhury A, Nelson LD, Teo MT, et al. MRE11 expression is predictive of cause-specific survival following radical radiotherapy for muscle-invasive bladder cancer. Cancer Res 2010;70:7017-26.

6. Laurberg JR, Brems-Eskildsen AS, Nordentoft I, et al. Expression of TIP60 (tat-interactive protein) and MRE11 (meiotic recombination 11 homolog) predict treatmentspecific outcome of localised invasive bladder cancer. BJU Int 2012;110:E1228-36.

7. Sakano S, Wada T, Matsumoto H, et al. Single nucleotide polymorphisms in DNA repair genes might be prognostic factors in muscle-invasive bladder cancer patients treated with chemoradiotherapy. Br J Cancer 2006;95:561-70.

8. Sak SC, Harnden P, Johnston CF, et al. APE1 and XRCC1 protein expression levels predict cancer-specific survival following radical radiotherapy in bladder cancer. Clin Cancer Res 2005;11:6205-11.

9. Walker AK, Karaszi K, Valentine H, et al. MRE11 as a Predictive Biomarker of Outcome After Radiation Therapy in Bladder Cancer. Int J Radiat Oncol Biol Phys 2019;104:809-18.

10. Stracker TH, Petrini JH. The MRE11 complex: starting from the ends. Nat Rev Mol Cell Biol 2011;12:90-103.

11. Vignard J, Mirey G, Salles B. Ionizing-radiation induced
DNA double-strand breaks: a direct and indirect lighting up. Radiother Oncol 2013;108:362-9.

12. Desai NB, Scott SN, Zabor EC, et al. Genomic characterization of response to chemoradiation in urothelial bladder cancer. Cancer 2016;122:3715-23.

13. Mitin T, Choudhury A. The role of biomarkers in bladder preservation management of muscle-invasive bladder cancer. World J Urol 2019;37:1767-72.

14. Magliocco AM, Moughan J, Simko J, et al. The impact of MRE11 in nuclear to cytoplasmic ratio on outcomes in muscle invasive bladder cancer an analysis of NRG/RTOG 8802, 8903, 9506, 9706, 9906, and 0233. J Clin Oncol 2017;25:343.

15. Martin RM, Kerr M, Teo MT, et al. Post-transcriptional regulation of MRE11 expression in muscle-invasive bladder tumours. Oncotarget 2014;5:993-1003.

16. Teo MT, Dyrskjøt L, Nsengimana J, et al. Next-generation sequencing identifies germline MRE11A variants as markers of radiotherapy outcomes in muscle-invasive bladder cancer. Ann Oncol 2014;25:877-83.

17. Van Allen EM, Mouw KW, Kim P, et al. Somatic ERCC2 mutations correlate with cisplatin sensitivity in muscle-invasive urothelial carcinoma. Cancer Discov 2014;4:1140-53.

18. Li Q, Damish AW, Frazier Z, et al. ERCC2 Helicase Domain Mutations Confer Nucleotide Excision Repair Deficiency and Drive Cisplatin Sensitivity in MuscleInvasive Bladder Cancer. Clin Cancer Res 2019;25:977-88.

19. Plimack ER, Dunbrack RL, Brennan TA, et al. Defects in DNA Repair Genes Predict Response to Neoadjuvant Cisplatin-based Chemotherapy in Muscle-invasive Bladder Cancer. Eur Urol 2015;68:959-67.

20. Abbosh PH, Plimack ER. Molecular and Clinical Insights into the Role and Significance of Mutated DNA Repair Genes in Bladder Cancer. Bladder Cancer 2018;4:9-18.

21. Eswara JR, Efstathiou JA, Heney NM, et al. Complications and long-term results of salvage cystectomy after failed bladder sparing therapy for muscle invasive bladder cancer. J Urol 2012;187:463-8.

22. Gray PJ, Fedewa SA, Shipley WU, et al. Use of potentially curative therapies for muscle-invasive bladder cancer in the United States: results from the National Cancer Data Base. Eur Urol 2013;63:823-9.

Cite this article as: Desai NB, Bagrodia A. The challenge of matching assays to biology in DNA damage response biomarkers for response to radiotherapy in bladder cancer. Transl Androl Urol 2019;8(Suppl 5):S514-S516. doi: 10.21037/tau.2019.07.05 